\title{
Avaliação do tempo de dreno de sucção em pacientes submetidos à artrodese lombar: estudo prospectivo randomizado
}

\author{
Ana Carolina Xavier Milagre ${ }^{1}$, Antonio Haddad Tápias Filho', Charbel Jacob Júnior², \\ Deborah Frigini Scardua1, Igor Machado Cardoso², José Lucas Batista Júnior², \\ Rodrigo Rezende ${ }^{3}$
}

Hospital Santa Casa de Misericórdia de Vitória, Vitória, ES, Brasil.

\section{RESUMO}

Objetivo: Avaliar o tempo mais adequado para o uso do dreno de sucção no pós-operatório de artrodese lombar. Métodos: Estudo descritivo, comparativo, randomizado, com uma amostra de 40 pacientes, dos quais 20 utilizaram o dreno de sucção por três dias de pós-operatório e outros 20 utilizaram o dreno de sucção por cinco dias de pós-operatório. Foram avaliadas e comparadas as complicações surgidas no pós-operatório dos pacientes de ambos os grupos e a Escala Visual Analógica da dor. As complicações avaliadas foram seroma, infecção superficial e deiscência de sutura. Resultados: Entre os 40 pacientes avaliados, encontramos $10 \%$ de complicações da ferida operatória no pós-operatório, sendo as mais frequentes o seroma e a infecção superficial, não havendo necessidade de reintervenção cirúrgica. Conclusão: Apesar de a utilização do dreno de sucção por cinco dias ter levado a um menor número de complicações e a um menor índice de dor no pós-operatório, esta não foi estatisticamente significante quando comparada com o grupo em que o dreno foi retirado no terceiro dia de pós-operatório.

\section{PALAVRAS-CHAVE}

Artrodese, região lombossacral, fusão vertebral, drenagem, cuidados pós-operatórios, descompressão cirúrgica, seroma.

\section{ABSTRACT}

Evaluation of the suction drain time in patients undergoing lumbar arthrodesis: prospective randomized study

Objective: Evaluation of the most appropriate time to use suction drain in the postoperative period of lumbar arthrodesis. Methods: A descriptive, comparative and randomized study, with a sample of 40 patients, 20 used the suction drain during three days of the postoperative and 20 used the suction drain during five days of the postoperative. The complications and the Visual Analogue Pain Scale were evaluated and compared in postoperative patients of both groups. The complications included seroma, superficial infection and wound dehiscence. Results: Among the 40 patients, we found $10 \%$ of wound complications in the postoperative period, the most common being seroma and superficial infection, with no need for surgical intervention. Conclusion: Although the use of the suction drain for five days has led to fewer complications and a lower rate of postoperative pain, this was not statistically significant when compared with the group where the drain was removed on the third day of the postoperative.

\section{KEYWORDS}

Arthrodesis, lumbosacral region, spinal fusion, drainage, postoperative care, decompression surgical, seroma.

1 Graduando do Curso de Medicina da Escola Superior de Ciências da Saúde da Santa Casa de Misericórdia (Emescam), Vitória, ES, Brasil.

2 Médico-assistente do Grupo de Coluna Vertebral do Hospital Santa Casa de Misericórdia de Vitória, Vitória, ES, Brasil.

3 Grupo de Coluna Vertebral do Hospital Santa Casa de Misericórdia de Vitória, Vitória, ES, Brasil. (in memoriam) 


\section{Introdução}

A artrodese é um procedimento amplamente realizado no tratamento cirúrgico de diversas patologias vertebrais, e sua indicação tem aumentado exponencialmente nas últimas duas décadas. ${ }^{1,2}$ Sua indicação ocorre principalmente nos casos de lombociatalgia refratária ao tratamento conservador adequado, em que nas radiografias dinâmicas se observam sinais de instabilidade segmentar vertebral associada ou não ao colapso do espaço discal observado na ressonância magnética, ${ }^{3-5}$ sendo o seu objetivo o alívio do quadro álgico incapacitante, gerado pelas doenças degenerativas. ${ }^{3}$

A técnica de artrodese vertebral mais frequentemente utilizada atualmente é a artrodese intersomática, que consiste na fusão tanto posterior quanto anterior, elevando, dessa maneira, a taxa de fusão vertebral. Embora essa técnica seja a de melhor resultado clínico e a mais utilizada, ela não é isenta de complicações, sendo infecção, seroma, lesão neurológica e pseudoartrose as mais frequentes. ${ }^{6-9}$

Sabe-se que a utilização do dreno de sucção no pós-operatório de cirurgia da coluna pode, muitas vezes, ser decisiva na redução do risco dessas complicações, que podem variar desde as mais simples, como seromas, até as mais temidas pelos cirurgiões, que são as compressões medulares causadas pelos hematomas epidurais. ${ }^{10,11}$ Portanto, a utilização do dreno de sucção é preconizada em cirurgias com grande descolamento tecidual e quando há extensa ressecção. Seu mecanismo de ação funciona por pressão negativa, e essa aspiração contínua previne o acúmulo de coleções e a formação de coágulos no espaço de drenagem ou no interior do próprio dreno.

A utilização do dreno de sucção em cirurgia de coluna já é bem estabelecida pela literatura, porém não está determinado qual o melhor momento para se retirar o dreno de sucção com máxima segurança, pois que sua retirada precoce pode resultar em seromas e hematomas e sua retirada tardia resulta em aumento da taxa de infecção. ${ }^{12-17}$ Portanto, a finalidade de nosso trabalho é tentar esclarecer essa dúvida existente na literatura, contribuindo sobremaneira com o resultado pós-operatório das cirurgias de coluna.

\section{Material e métodos}

Foi realizado um estudo prospectivo e randomizado no Hospital Santa Casa de Misericórdia de Vitória (HSCMV), no qual se avaliaram 40 pacientes com diagnóstico de estenose de canal lombar secundária à doença discal degenerativa, sendo esses submetidos à descompressão do canal medular associada à artrodese posterolateral ou intersomática de até dois níveis lombares.

No pré-operatório, foram documentados a idade, 0 sexo e o diagnóstico, sendo realizada uma randomização dos pacientes, por meio de um sorteio realizado por um integrante da equipe cirúrgica que não está entre os autores do trabalho. Havia 40 cartões em uma urna, sendo 20 com o número 1 e 20 com o número 2 . Após cada sorteio, o cartão retirado da urna foi descartado. Nos pacientes do grupo 1, o dreno foi utilizado no pós-operatório por 72 horas (três dias), enquanto nos do grupo 2 foi utilizado por 120 horas (cinco dias), não se considerando o volume drenado para a retirada desse dreno. Independentemente do resultado do sorteio, todos os pacientes receberam alta hospitalar no quinto dia de pós-operatório.

No pós-operatório, utilizou-se a Escala Visual Analógica (EVA) como forma de mensuração da dor na ferida operatória e analisou-se a presença de complicações no sítio cirúrgico como seroma, infecção superficial e deiscência da ferida operatória no momento da retirada do dreno, no dia da alta, após 10 a 15 dias da alta (primeiro retorno) e após três a quatro semanas do último retorno (segundo retorno). Para manejo da dor no pós-operatório, foi utilizada uma analgesia-padrão para todos os pacientes com anti-inflamatórios e opioides.

O critério de inclusão foi pacientes com quadro de lombociatalgia associada à claudicação neurogênica secundária à estenose de canal lombar, sem melhora com no mínimo 20 sessões de fisioterapia adequada, em que se visualizaram na radiografia lombar dinâmica sinais de instabilidade em até dois níveis vertebrais.

O critério de exclusão foi pacientes com instabilidades em mais de dois níveis, ou artrodese em outros segmentos vertebrais, sem sinais de instabilidade lombar na radiografia dinâmica, em que se optou pela discectomia simples.

Para análise do índice de complicações surgidas no pós-operatório de cada grupo, utilizamos o teste de qui-quadrado, e para análise da dor no pós-operatório em cada grupo, utilizamos o teste não paramétrico de Friedman na retirada do dreno, na alta hospitalar, entre 10 e 15 dias (primeiro retorno) e após três a quatro semanas do último retorno (segundo retorno).

\section{Resultados}

Foram avaliados 40 pacientes, sendo 15 do sexo masculino, com média de idade de 40 anos, mínimo de 30 anos e máximo de 65 anos. Nesse total de pacientes, encontramos quatro complicações da ferida operatória 
no pós-operatório, sendo as mais frequentes o seroma e a infecção superficial. Apesar das complicações citadas, em nenhuma houve necessidade de reintervenção cirúrgica.

Ao analisarmos separadamente as complicações nos dois grupos, encontramos que no grupo em que o dreno permaneceu por três dias (grupo 1) houve dois seromas e uma infecção superficial, enquanto no grupo em que o dreno permaneceu cinco dias (grupo 2) ocorreu apenas uma complicação, que consistiu em um seroma (Tabela 1).

Ao realizarmos a análise estatística, utilizamos o teste de qui-quadrado, por se tratar do mais indicado para nossos resultados, o qual indicou que não há associação entre os períodos de retirada do dreno (terceiro e quinto dia) e a existência de complicações, tanto para o grupo observado entre 10 e 15 dias da alta hospitalar (primeiro retorno), com p-valor de 0,292 , quanto para o grupo de três a quatro semanas do último retorno (segundo retorno), com p-valor de 0,756.

Em relação à dor no pós-operatório, a média da Escala Visual Analógica (EVA), em geral, foi semelhante nos dois grupos, embora tenhamos observado grande diminuição da EVA na última avaliação realizada com três a quatro semanas no grupo 2 (Tabela 2).

Utilizamos o teste não paramétrico de Friedman para análise estatística dos resultados referentes à dor pós-operatória nos dois grupos, e ele revelou que não houve diferença significativa para a retirada do dreno no terceiro ou no quinto dia e tampouco quando considerados esses dois grupos juntos, com p-valor de 0,711 , 0,229 e 0,380 , respectivamente.

\section{Discussão}

Diversos fatores interferem direta ou indiretamente no resultado da cirurgia de coluna. Um importante fator a ser considerado é o tempo de utilização do dreno de sucção no período pós-operatório, sendo as possíveis complicações de sua não utilização extremamente prejudiciais para a cirurgia, como surgimento de hematomas epidurais, deiscência de sutura e, frequentemente, seromas que podem aumentar a taxa de infecção do sítio cirúrgico. ${ }^{10,11}$ Por outro lado, a sua utilização prolongada acarreta aumento do tempo de internação, maior sangramento e, consequentemente, maior número de transfusões sanguíneas. ${ }^{18}$ Portanto, sabemos da importância da utilização do dreno no pós-operatório da cirurgia de coluna lombar, porém o que se discute na literatura é qual o melhor momento para sua retirada, pois tanto a retirada precoce como a tardia podem levar a complicações indesejadas.

Alguns autores utilizam como critério de retirada do dreno o volume de drenagem em 24 horas. ${ }^{10,18}$ Um dos problemas da utilização desse método é que alguns pacientes continuam drenando grande volume mesmo após um longo tempo de pós-operatório, e a manutenção do dreno nesses casos poderia facilitar o aparecimento de complicações, como infecção do sítio cirúrgico. Em nosso trabalho, desconsideramos o volume drenado e adotamos como critério único o tempo pós-operatório. Não encontramos na literatura nenhum trabalho semelhante ao nosso, em que se analisaram as complicações pós-operatórias em dois grupos de pacientes independentes que realizaram a mesma

\begin{tabular}{|c|c|c|c|}
\hline & Grupo 1 (3 dias) & Grupo 2 (5 dias) & Total \\
\hline Total de pacientes & 20 & 20 & 40 \\
\hline Seroma & $2(10 \%)$ & $1(5 \%)$ & $3(7,5 \%)$ \\
\hline Infecção superficial & $1(5 \%)$ & 0 & $1(2,5 \%)$ \\
\hline Deiscência & 0 & 0 & 0 \\
\hline Deiscência com secreção purulenta & 0 & 0 & 0 \\
\hline Total de complicações & $3(15 \%)$ & $1(5 \%)$ & $4(10 \%)$ \\
\hline
\end{tabular}

\begin{tabular}{lccc}
\hline & Tabela 2 - Comparações da intensidade de dor (EVA) entre os períodos de três e cinco dias & \\
\hline & Grupo 1 (EVA) & Grupo 2 (EVA) & \multicolumn{1}{c}{ Significância p } \\
\hline Na retirada do dreno & 0,3 & 0,55 & 0,55 \\
Na alta & 0,5 & 0,738 \\
Entre 10 e 15 dias & 0,55 & 0,85 & 0,799 \\
Entre 3 e 4 semanas & 0,55 & 0,947 \\
Total & 0,475 & 0,537 \\
\hline
\end{tabular}


cirurgia, sendo a diferença entre os grupos apenas o tempo de utilização do dreno.

Kanayama et al. ${ }^{10}$ e Brown e Brookfield, ${ }^{12}$ em seus estudos, compararam dois grupos de pacientes; em um utilizaram o dreno de sucção e no outro não utilizaram o dreno em nenhum momento no pós-operatório. No trabalho de Brown e Brookfield, ${ }^{12}$ apenas se observou aumento de temperatura da ferida pós-operatória no grupo que não utilizou o dreno, não sendo observada diferença entre os dois grupos quanto a infecção e hematoma epidural. Já no trabalho de Kanayama et al., ${ }^{10}$ houve maior taxa de hematoma pós-operatório no grupo que utilizou o dreno, porém tal fato não influenciou no resultado do tratamento.

Em nosso estudo, observamos maior taxa de complicações nos pacientes que utilizaram o dreno por menor tempo, sendo mais frequentes os casos de seroma que, apesar de não colocarem em risco o procedimento cirúrgico realizado, acarretam uma série de preocupações tanto para a equipe médica como para o paciente e seus familiares. Julgamos que a maior presença de tais complicações tenha acontecido devido ao fato de não ter ocorrido tempo suficiente para o fechamento adequado da ferida operatória.

Aono et al. ${ }^{19}$ encontraram nos resultados de seu estudo que metade dos pacientes submetidos à cirurgia de coluna lombar apresentou sintomas, como dor nos membros inferiores ou disfunção vesical, relacionados à formação de hematoma epidural após a remoção do dreno de sucção, utilizado por pelo menos 48 horas após a cirurgia. Observamos em nosso trabalho que, no grupo em que o dreno foi utilizado por tempo mais prolongado, a média geral do EVA foi menor, principalmente após três a quatro semanas de pós-operatório.

Apesar de a necessidade de utilização do dreno de sucção no pós-operatório encontrar-se bem estabelecida na literatura, a dúvida sobre qual é o melhor momento para sua retirada ainda permanece. Julgamos que ainda devemos levar em consideração diversos fatores, como o volume drenado, as condições locais da ferida e o tempo de utilização do dreno de sucção, para a decisão sobre qual o melhor momento para interromper a drenagem no pós-operatório.

\section{Conclusão}

Apesar de a utilização do dreno de sucção por cinco dias ter levado a um menor número de complicações e a um menor índice de dor no pós-operatório, esta não foi estatisticamente significante quando comparada com o grupo em que o dreno foi retirado no terceiro dia de pós-operatório.

\section{Conflito de interesses}

Os autores declaram a inexistência de conflito de interesses na realização deste trabalho.

\section{Referências}

1. Olivares LMR, Vaca JC, Martínes VPM, Aguirre AA, ReyesSánches AA. Desarrollo de enfermedad del segmento adyacente em arthrodesis circunferencial lumbar: cuatro años de seguimiento. Coluna/Columna. 2006;5(1):13-8.

2. Gillet P. The fate of the adjacent motion segments after lumbar fusion. J Spinal Disord Tech. 2003;16(4):338-45.

3. Vaccaro AR, Betz RR, Zeidman SM. Cirurgia da coluna: princípios e prática. $2^{\mathrm{a}}$ ed. Rio de Janeiro: Di Livros; 2007.

4. Avanzi O, Chih LY, Meves R, Silber MF. Tratamento da instabilidade lombar com parafusos pediculares. Acta Ortop Bras. 2005;13(1):5-8.

5. Kim TY, Kang KT, Yoon DH, Shin HC, Kim KN, Yi S, et al. Effects of lumbar arthrodesis on adjacent segments: differences between surgical techniques. Spine (Phila Pa 1976). 2012;37(17):1456-62.

6. Di Lauro L, Poli R, Bortoluzzi M, Marini G. Paresthesias after lumbar disc removal and their relationship to epidural hematoma. Report of two cases. J Neurosurg. 1982;57(1):135-6.

7. Lawton MT, Porter RW, Heiserman JE, Jacobowitz R, Sonntag VK, Dickman CA. Surgical management of spinal epidural hematoma: relationship between surgical timing and neurological outcome. J Neurosurg. 1995;83(1):1-7.

8. Morse $\mathrm{K}$, Weight M, Molinari R. Extensive postoperative epidural hematoma after full anticoagulation: case report and review of the literature. J Spinal Cord Med. 2007;30(3):282-7.

9. Koutsoumbelis S, Hughes AP, Girardi FP, Cammisa FP Jr, Finerty EA, Nguyen JT, et al. Risk factors for postoperative infection following posterior lumbar instrumented arthrodesis. J Bone Joint Surg Am. 2011;93(17):1627-33.

10. Kanayama M, Oha F, Togawa D, Shigenobu K, Hashimoto $\mathrm{T}$. Is closed-suction drainage necessary for single-level lumbar decompression?: review of 560 cases. Clin Orthop Relat Res. 2010;468(10):2690-4.

11. Khan MH, Rihn J, Steele G, Davis R, Donaldson WF 3rd, Kang JD, et al. Postoperative management protocol for incidental dural tears during degenerative lumbar spine surgery: a review of 3,183 consecutive degenerative lumbar cases. Spine (Phila Pa 1976). 2006;31(22):2609-13.

12. Brown MD, Brookfield KF. A randomized study of closed wound suction drainage for extensive lumbar spine surgery. Spine (Phila Pa 1976). 2004;29(10):1066-8.

13. Payne DH, Fischgrund JS, Herkowitz HN, Barry RL, Kurz LT, Montgomery DM. Efficacy of closed wound suction drainage after single-level lumbar laminectomy. J Spinal Disord. 1996;9(5):401-3.

14. Beer KJ, Lombardi AV Jr, Mallory TH, Vaughn BK. The efficacy of suction drains after routine total joint arthroplasty. J Bone Joint Surg Am. 1991;73(4):584-7.

15. Ritter MA, Keating EM, Faris PM. Closed wound drainage in total hip or total knee replacement. A prospective, randomized study. J Bone Joint Surg Am. 1994;76(1):35-8.

16. Cobb JP. Why use drains? J Bone Joint Surg Br. 1990;72(6):993-5. 
17. Scuderi GJ, Brusovanik GV, Fitzhenry LN, Vaccaro AR. Is wound drainage necessary after lumbar spinal fusion surgery? Med Sci Monit. 2005;11(2):CR64-6.

18. Diab M, Smucny M, Dormans JP, Erickson MA, Ibrahim K, Lenke LG, et al. Use and outcomes of wound drain in spinal fusion for adolescent idiopathic scoliosis. Spine (Phila Pa 1976). 2012;37(11):966-73.

19. Aono H, Ohwada T, Hosono N, Tobimatsu H, Ariga K, Fuji $\mathrm{T}$, et al. Incidence of postoperative symptomatic epidural hematoma in spinal decompression surgery. $\mathrm{J}$ Neurosurg Spine. 2011;15(2):202-5.
Endereço para correspondência

Charbel Jacob Júnior

Serviço de Coluna Vertebral do

Hospital Santa Casa de Misericórdia de Vitória

Rua Doutor João Santos Neves, 143, Vila Rubim

29018-180 - Vitória, ES, Brasil

E-mail: Jcharbel@gmail.com 